\title{
Strain Elastography (SE) for liver fibrosis estimation - which elastic score to calculate?
}

\author{
Mariana M. Gersak ${ }^{1}$, Monica Lupșor-Platon ${ }^{2}$, Radu Badea ${ }^{2}$, Anca Ciurea ${ }^{1}$, Sorin M. Dudea ${ }^{1}$
}

${ }^{1}$ Department of Radiology, ${ }^{2}$ Department of Medical Imaging, Regional Institute of Gastroenterology and Hepathology

"Prof. Dr. Octavian Fodor”, "Iuliu Hațieganu” University of Medicine and Pharmacy, Cluj-Napoca, Romania

\begin{abstract}
Liver fibrosis scoring by liver biopsy has become a rarity in daily practice mainly because many non-invasive methods with similar accuracy have been developed. Among all ultrasound elastography imaging methods, Strain Elastography (SE) is the most widely available. Although SE is a qualitative and semi-quantitative method, there is reliable applicability for liver fibrosis estimation and multiple ways to transform SE into a quantitative method, in order to obtain a fibrosis score. The aim of this review is to briefly introduce all these methods and to offer support in choosing the best estimation method for liver fibrosis, with SE.
\end{abstract}

Keywords: strain elastography, liver, fibrosis, elastic score

\section{Introduction}

The most common causes of liver fibrosis are viral, alcoholic, or non-alcoholic chronic hepatitis. The incidence of liver fibrosis is increasing due to the increased number of patients with diabetes, metabolic syndrome, and chronic viral hepatitis (B and C). Staging liver fibrosis subsequent to chronic viral hepatitis has a particular clinical importance, mainly because significant liver fibrosis (METAVIR score $\geq \mathrm{F} 2$ ) is an important criterion for recommending antiviral therapy $[1,2]$.

Liver biopsy is considered the reference method for the diagnosis and staging of liver fibrosis. It is well known that inter and intra observer variability of liver biopsy is relatively high $[3,4]$. False negative results have been reported in up to $30 \%$ of the cases $[3,4]$ complications were reported, some of them requiring medical care $(1-3 \%$ of

Received 29.07.2016 Accepted 10.09.2016

Med Ultrason

2016, Vol. 18, No 4, 481-487

Corresponding author: Monica Lupşor-Platon

Department of Medical Imaging,

"Prof. Dr. Octavian Fodor" Regional Institute

of Gastroenterology and Hepathology,

19-21 Croitorilor Street,

400162 Cluj-Napoca, Romania

E-mail: monica.lupsor@umfcluj.ro liver biopsy cases) [5].Up to $25 \%$ of the patients complained of post procedural local pain [5]. Non-invasive methods for predicting liver fibrosis, as an alternative to liver biopsy, were studied intensely. These methods comprise ultrasound elastography [transient elastography (TE), Virtual Touch Tissue Quantification (VTTQ)/ Acoustic Radiation Force Imaging (ARFI elastography), two dimensional Shear-Wave elastography (2D-SWE), strain elastography (SE)], magnetic resonance elastography (MR elastography)] and serological tests. Along with the development of non-invasive methods for liver stiffness estimation, the number of liver biopsies has dropped significantly, thus limiting the prospective studies due to the lack of a reference method.

Strain Elastography (SE) was the first type of commercially available elastography developed by Hitachi. At first, it was only available on linear probes [1]. This type of elastography displays a color-coded map, which depends on the degree of tissues compressibility. The color scale ranges from blue (stiff) to red (soft) [2].

RTE offers both a qualitative (color- coded map) and a semi-quantitative elasticity measurement of liver stiffness (by computing the Strain Ratio- SR). SE assesses relative tissue stiffness by comparing surrounding tissues in terms of elasticity. Various groups explored the use of mathematical formulas, in the attempt to obtain a numeri- 
cal indicator for the characterization and staging of liver fibrosis with SE.

Several methods for liver fibrosis quantification by interpreting and processing the color-coded SE maps displayed by the equipment were investigated. The aim of this paper is to provide an overview of these methods, and to analyze the performance of each method/score, for obtaining a reliable non-invasive liver stiffness estimation and fibrosis stage assessment.

\section{SE methods for liver fibrosis quantification}

1. Fujimoto et al [6] were among the first researchers who initiated the development of various ways to transform the color-coded maps generated by RTE, into an elastic score in order to quantify liver fibrosis [7]. Using Hitachi equipment with a linear probe, the ROI was placed immediately under the liver capsule, with the patient in apnea. The color patterns of RTE were interpreted and coded into four elastography scores (fig 1), one for each degree of fibrosis. The higher the score the lesser elasticity the more advanced fibrosis. The score was compared with other serological tests (ALT, total bilirubin, albumin, choline esterase, total cholesterol, prothrombin time, platelet count, and Type IV Collagen 7S), but among all of them, the elasticity score was correlated only with collagen IV type 7S [6].

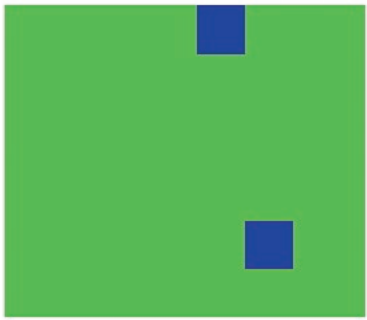

Score 1

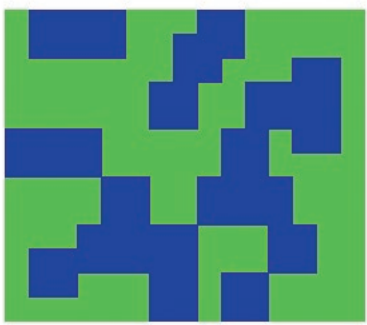

Score 3

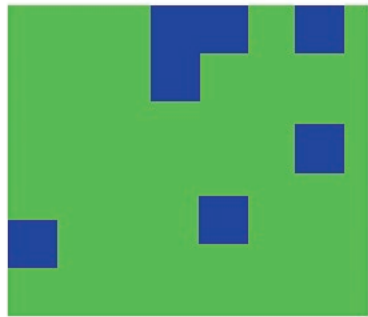

Score 2

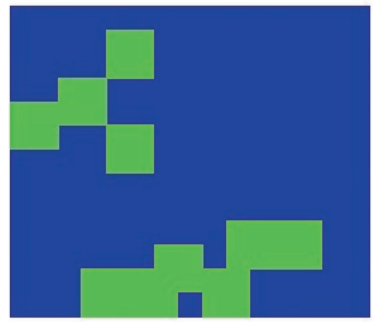

Score 4
Fig 1. Elasticity score according to Fujimoto et al [6]. Score 1 - the entire colored area is shown as relatively uniform, light green. Score 2 - Partially mottled blue regions are shown in the light green colored area. Score 3 - Light green and blue are mixed in the colored area (almost a fifty-fifty mix). Score $4-$ most of the colored area is blue [6].
The main disadvantage of this score is the subjectivity of the operator. Thus, Fujimoto et al attenuated this limit by using the opinion of 6 blinded operators who worked independently and the mean score was obtained for each patient, in order to establish the elasticity score. Unfortunately, inter observer variability was not mentioned in this study.

2. Elasticity score. Friedrich-Rust et al [1] proposed another approach to quantify liver fibrosis using RTE. Using Hitachi equipment and a linear probe they positioned the patients in supination, with the right hand above their head. The patients were asked to breathe with normal amplitude. The transducer was placed in a right intercostal space, where liver parenchyma had the thickness of at least $6 \mathrm{~cm}$, avoiding large intrahepatic vessels. Ten RTE images, with an approximate area of 350$500 \mathrm{~mm}^{2}$ were stored for each patient. These images were processed and used for the development of the elasticity score (ES) [1]. Initially, the formula for ES included only parameters from recorded images (see formula no 1 in appendix). Later on, to increase the accuracy of ES, the authors introduced also two biological parameters: the number of platelets and gamma-glutamyl transpeptidase (GGT) (see formula no 2 in appendix). Using the second ES formula, Se (Sensibility) and NPV (negative predictive value) were improved (Table I). Comparing RTE with TE, similar results were found for the detection of significant fibrosis $(\geq F 2)$, area under the curve (AUC) was 0.75 for ES and $0.75-0.84$ for TE [1,7-9].

Table I. Elasticity score (ES) according to Friederich et al 2007 [1].

\begin{tabular}{lllllll}
\hline Fibrosis stage & \multicolumn{2}{l}{ cutoff values } & Se & NPV & AUC \\
\hline & $\mathbf{F} \geq \mathbf{2}$ & $\mathbf{F} \geq \mathbf{F 3}$ & $\mathbf{F}=\mathbf{4}$ & $\mathbf{F} \geq \mathbf{2}$ & & \\
\cline { 2 - 7 } ES & 100.1 & 102.5 & 111.75 & 80 & 78.6 & - \\
$\begin{array}{l}\text { ES+ biologic } \\
\text { parameters }\end{array}$ & 99.1 & 99.7 & 114.7 & 84.4 & 87.3 & 0.75 \\
\hline
\end{tabular}

Se - sensibility; NPV - negative predictive value; AUC - area under the curve; $\mathrm{F}$ - fibrosis stage

3. The Liver fibrosis index (LFI) was first published by Tatsumi et al in 2008 [10], in a study involving 125 patients with viral hepatitis of various etiologies. To assess the stiffness of the left liver lobe, the probe was placed in the epigastrium, and for the right liver lobe, the probe was placed in a right intercostal space. The ROI was placed immediately under the liver capsule. Liver elastography was obtained by applying a minimal external compression on the probe, associated with the transmitted hepatic compression from each heartbeat. In order to obtain LFI, the color map was coded from 0 (blue) to 255 (red). Then, a few parameters were calculated: mean, 
standard deviation (SD), percentage of blue area in the ROI (\%AREA), complexity $(\mathrm{COMP}=$ length squared divided by blue area) and inverse difference moment (IDM). The complete formula of LFI was not published but the authors found a negative correlation between LFI and the fibrosis stage estimated by TE $(\mathrm{r}=-0.587, \mathrm{p}<0.05)$.

Later, the same authors, conducted another study on a group of 44 patients with different degrees of fibrosis, induced by chronic hepatitis $\mathrm{C}$. The authors improved the formula by adding 4 new parameters for LFI: kurtosis of strain histogram (KURT), skewness of strain histogram (SKEW), entropy (ENT) and angular second moment (ASM) [11]. They placed the probe in the right intercostal space and recorded 6 RTE images. The authors reported that LFI increased with the degree of fibrosis, and statistically significant differences were identified for liver fibrosis stage F1/F2, F2/F3 and F2/F4 $(p<0.05)$ but not for F3/F4. RTE examination could not be performed in patients with ascites, thick subcutaneous tissue, liver atrophy and narrow intercostal spaces, where TE displayed also a limited performance. Unfortunately, the new formula was not published.

Ferraioli et al [12] used the same parameters to calculate LFI as Tatsumi et al. In their study, the ROI was placed $1 \mathrm{~cm}$ under the liver capsule, avoiding the large vessels (fig 2). There was no external compression exerted on the transducer, heartbeat being sufficient for RTE acquisition. The image was considered valid when the box was completely colored inside and no artifacts were observed.

The adjusted formula of the LFI was obtained by averaging the obtained parameters for 5 images (see formula no 3 in appendix). The obtained cutoff values, Se, Sp and AUC which are presented in Table II [12].

Fujimoto et al [13] included a larger number of patients in their study (295 patients) and they published another LFI formula, based on the same 9 parameters suggested by Tatsumi et al (see formula no 4 in appendix) [13]. In this study, LFI was correlated with liver fibrosis $(\mathrm{r}=0.68, \mathrm{p}<0.001)$, and clear differences were found either F0-F1 and F2-F4 or F0-F3 and F4 (Table II)
Yada et al [14], in a multicentric study including 245 patients with chronic viral hepatitis B and C, calculated LFI by analyzing the 9 parameters and averaging the results from 10 valid acquired images in a similar fashion and the same formula which Fujimoto et al used in their study [13]. Although there were no significant differences between stages F2 and F3, there were significant differences between advanced fibrosis and cirrhosis. The AUROC for $\mathrm{F} 4$ and $\geq \mathrm{F} 2$ was higher than in the case of serological markers (AUROC of 0.865 and 0.846 for LFI vs 0.789 and 0.809 for aspartate aminotransferase-toplatelet ratio index (APRI) [14].

Tomeno et al measured LFI in the same fashion as Fujimoto et al, in a study group of patients with chronic hepatitis and non-alcoholic fatty liver disease (NAFLD) [15]. There was a correlation between LFI and liver fibrosis in patients with chronic hepatitis $(\mathrm{r}=0.319, \mathrm{p}=0.0009)$ but not in patients with NAFLD $(r=0.267, \mathrm{p}=0.179)$ [15].

Meng et al [16] improved the LFI formula, by introducing CONT (contrast) and CORR (correlation) as parameters, in a sample of chronic hepatitis type B. This way, they obtained significant differences between F1 and F2 and between F3 and F4 with AUCs $\geq 0.75$ (Table III). They obtained similar results in discriminating liver fibrosis with both TE and RTE. The cutoff values for LFI are described in table II. Unfortunately, the formula was not given, most probably because by the time the study was published,

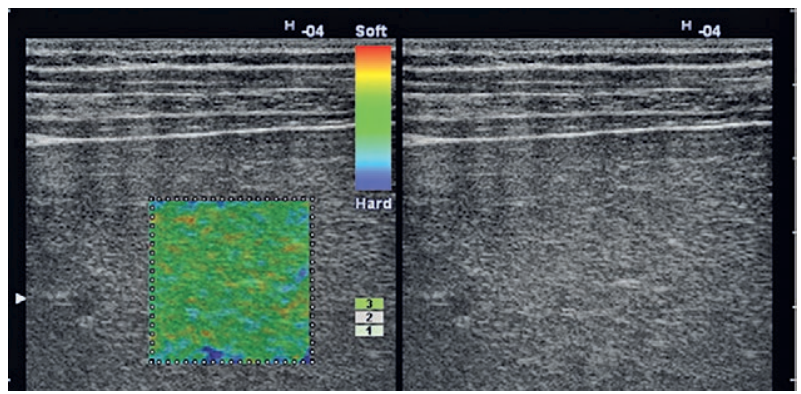

Fig 2. Real time elastography acquisition for liver fibrosis index computation, according to Ferraioli et al [12]. The ROI is placed at least $1 \mathrm{~cm}$ under the liver capsule.

Table II. Liver fibrosis index (LFI) cutoff values and diagnostic usefulness

\begin{tabular}{|c|c|c|c|c|c|c|c|c|c|c|c|c|}
\hline Authors & Cuto & & & AUC & & & $\mathrm{Se}$ & & & Sp & & \\
\hline & $F \geq 2$ & $\mathrm{~F} \geq \mathrm{F3}$ & $F=4$ & $F \geq 2$ & $F \geq F 3$ & $F=4$ & $F \geq 2$ & $\mathrm{~F} \geq \mathrm{F} 3$ & $F=4$ & $F \geq 2$ & $\mathrm{~F} \geq \mathrm{F3}$ & $F=4$ \\
\hline $\begin{array}{l}\text { Ferraiolli } \\
2010 \text { [12] }\end{array}$ & 1.82 & 1.86 & 2.33 & 0.74 & 0.80 & 0.80 & 81.7 & 91.7 & 66,7 & 60 & 57.5 & 84 \\
\hline $\begin{array}{l}\text { Fujimoto } \\
2013 \text { [13] }\end{array}$ & 1.92 & - & 2.56 & 0.82 & - & 0.87 & 78.6 & - & - & 78 & - & 80.5 \\
\hline $\begin{array}{l}\text { Yada } \\
2013 \text { [14] }\end{array}$ & 2.05 & 2.28 & 2.36 & 0.8 & 0.87 & 0.85 & 70 & 78.4 & 73,5 & 76.4 & 80.2 & 79.7 \\
\hline $\begin{array}{l}\text { Meng } \\
2015[16]\end{array}$ & 3.73 & 3.89 & 4.62 & 0.88 & 0.868 & 0.752 & 88.1 & 90 & 80.1 & 74.7 & 78 & 76.2 \\
\hline
\end{tabular}

$\mathrm{Se}$ - sensibility; Sp - specificity; AUC - area under the curve; F - fibrosis stage 
Hitachi already had implemented the software which performs the LFI automatically after the ROI is positioned.

In the same manner, Hu et al used the LFI calculus, containing all the 11 elements above described by Meng et al, on a sample of healthy volunteers, with normal BMI (body mass index). He obtained a mean LFI value $1.31 \pm$ 0.25 , for healthy volunteers. They also compared the normal values with a sample of patients with liver fibrosis and found a significant difference between F0 and F1-F2 $(\mathrm{p}<0$.001). The optimal liver fibrosis index threshold value for discriminating normal liver from non-cirrhotic chronic liver disease was 2.12 [17].

Subsequently, other authors have used strain ratio (SR) for assessing liver fibrosis, performing a ratio between the two areas of interest (ROI): one placed on the liver tissue and the other one in the intrahepatic veins [18-20], or in the intercostal muscles [21-23].

4. Elastic ratio (ER1) was studied by Kanamoto et al [21], in a study of 41 patients with various pathologies. They performed a SR between hepatic parenchyma and intercostal muscles (fig 3). They used a Hitachi ultrasound machine and linear transducer which had attached a device which applied a constant compression and relaxation on the patient's skin. ER1 was measured as the strain ratio between liver parenchyma and intercostal muscles, with rectangular ROIs. A significant relation between the stage of fibrosis and ER1 was identified $(\mathrm{p}<0.0001)$ [21]. The median ER1 are described in table III.

Later, ER1 was used by Xie et al [23] for the evaluation of 71 patients with hepatitis $\mathrm{B}$, in a similar manner to Kanamoto et al. They used similar criteria of staging chronic hepatitis, according to the Chinese Program of Prevention and Cure for Viral Hepatitis (S). The median ER1 for liver fibrosis are detailed in table III. Significant differences were identified between stage $\mathrm{S} 0 / \mathrm{S} 1, \mathrm{~S} 1 / \mathrm{S} 2$, $\mathrm{S} 2 / \mathrm{S} 3, \mathrm{~S} 3 / \mathrm{S} 4$, and $\mathrm{S} 0 / \mathrm{S} 4(\mathrm{p}<0.001)$ [23].

Altiparmak et al [22] calculated ER1 in a study of 59 patients with acute-chronic liver disease. They used a Toshiba ultrasound and a linear transducer. The ROIs were oval, placed in the intercostal muscle and in the liver parenchyma, respectively. A manual, external com-

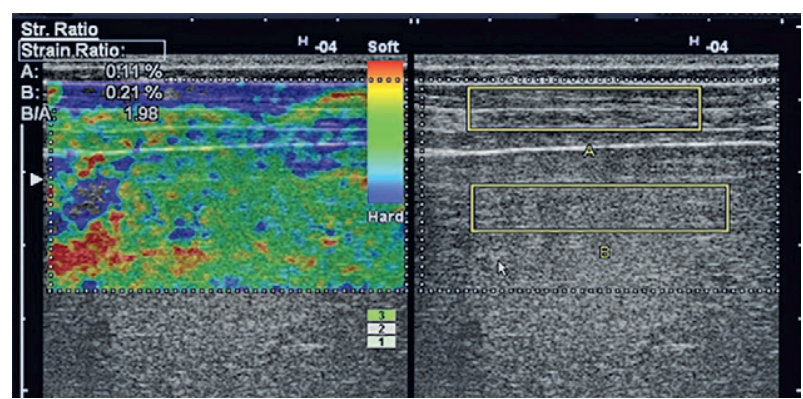

Fig 3. Elastic ratio between liver parenchyma (B) and intercostal muscles (A), according to Kanamoto et al [21].

pression-decompression was applied on the transducer and the patients were asked to maintain apnea after a deep inspiration till the acquisition was performed [22]. It is worth noting that, in this study, ER1 increased proportionally with fibrosis stage - contrary to other studies. This is most probably because these authors used a Toshiba ultrasound which calculates the ratio in a different manner than Hitachi, regarding the nominator and the denominator of the ratio.

The cutoff values, Se, Sp and AUC of ER1 are detailed in table III.

5. Elastic ratio (ER2) was studied by Koizumi et al [18], as representing the SR between small intrahepatic veins (with a caliber $<3 \mathrm{~mm}$ ) and liver parenchyma (fig 4). The small intrahepatic veins were preferred for SR2 calculus because the elasticity of portal vein might have been influenced by liver stiffness and diseases such as arteriosclerosis. For each patient, RTE was performed in 4 different areas by two examiners, in expiration or in inspiration. For every area the ER2 was calculated on 5 images. The ROI was placed within $1 \mathrm{~cm}$ of the liver capsule, and the dimensions were set at $0.3 / 0.5 \mathrm{~cm}$ for intrahepatic veins and $2 / 1 \mathrm{~cm}$ for liver parenchyma. The authors observed that the manual external compression on the transducer is not necessary, heart beats being sufficient for the RTE acquisition, with a good inter- and intra-observer variability [18]. In patients who could not be properly evaluated by gray scale ultrasonography, RTE was also limited (e.g. thick subcutaneous tissue, postop-

Table III. Elastic Ratio 1 (ER1) cutoff values and diagnostic usefulness

\begin{tabular}{|c|c|c|c|c|c|c|c|c|c|c|c|c|}
\hline \multirow[t]{2}{*}{ Authors } & \multicolumn{3}{|c|}{ Cutoff } & \multicolumn{3}{|c|}{ AUC } & \multicolumn{3}{|l|}{$\mathrm{Se}$} & \multicolumn{3}{|l|}{ Sp } \\
\hline & $F \geq 2$ & $F \geq F 3$ & $F=4$ & $F \geq 2$ & $F \geq F 3$ & $F=4$ & $F \geq 2$ & $F \geq F 3$ & $F=4$ & $F \geq 2$ & $F \geq F 3$ & $F=4$ \\
\hline $\begin{array}{l}\text { Kanamoto } \\
2009 \text { [21] }\end{array}$ & 1.18 & 0.75 & 0.6 & - & 0.95 & - & 96.2 & 95.5 & 93.3 & 73.3 & 89.4 & 73.1 \\
\hline $\begin{array}{l}\text { Xie } \\
2012[23]^{*}\end{array}$ & 1.1 & 0.75 & 0.6 & 0.86 & 0.74 & 0.79 & 77.8 & 61.5 & 50 & 80 & 91.1 & 96.7 \\
\hline $\begin{array}{l}\text { Altiparmak } \\
2015[22]^{* *}\end{array}$ & 4.38 & $\begin{array}{l}4.57 \\
(\mathrm{~F}=3)\end{array}$ & - & 0.92 & $\begin{array}{l}0.76 \\
(F=3)\end{array}$ & - & 92 & $\begin{array}{l}80 \\
(\mathrm{~F}=3)\end{array}$ & - & 85.3 & $\begin{array}{l}66.7 \\
(F=3)\end{array}$ & - \\
\hline
\end{tabular}

$\mathrm{Se}$ - sensibility; $\mathrm{Sp}$ - specificity; AUC - area under the curve; F - fibrosis stage; $*$ The criteria for staging of chronic hepatitis was according to Chinese Program of Prevention and Cure for Viral Hepatitis (S); ** The ES was calculated using Toshiba ultrasound 


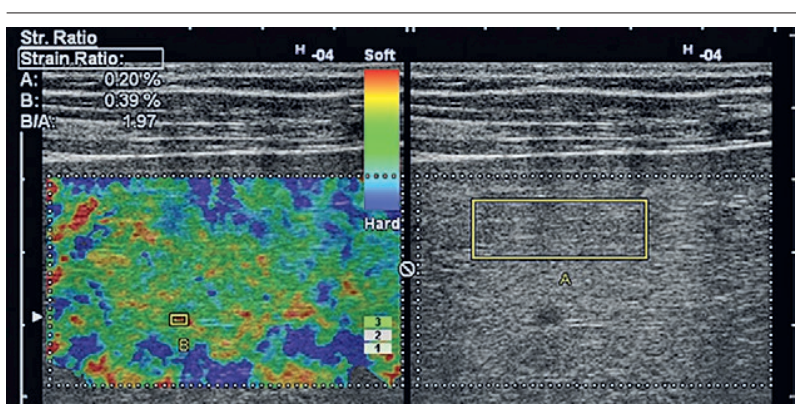

Fig 4. Elastic ratio between liver parenchyma (A) and small hepatic vein (B), according to Koizumi et al [18].

erative scars, uncooperative patients, liver atrophy and large amount of ascites) [18].

The obtained cutoff values are presented in table IV. There was a significant correlation between liver fibrosis stages and elastic ratio $(r=0.82, p<0.001)$. There were no significant differences between the mean ER2 values obtained in the 4 areas of measurement. Inter-observer variability was excellent in patients with a BMI $<25 \mathrm{~kg} / \mathrm{m}^{2}$ and, with subcutaneous tissue thickness less than $20 \mathrm{~mm}$. In patients over these values, inter-observer variability was slightly lower. In patients with BMI over $30 \mathrm{~kg} / \mathrm{m}^{2}$, ER2 could not be calculated [18].

The utility of ER2 was demonstrated also in patients with non-alcoholic fatty liver disease (NAFLD) [19]. ER2 accurately depicted $F \geq F 1, F \geq F 2, F \geq F 3$ and F4 (table IV) The same technique was used by $\mathrm{Hu}$ et al [20], who studied 96 patients with chronic hepatitis B and 45 volunteers. They showed a good reproducibility of ER2, with an intra-observer variability of 0.838 and inter-observer of $0.805(p<0.001)$. The cutoff values are presented in table IV.

Altiparmak et al [22] calculated also the ER2. They applied a manual compression-decompression on the transducer, and the patient was asked to keep apnea after a deep inspiration. This way, mean ER2 was $2.83 \pm 0.45$ for F0, $3.78 \pm 0.47$ for $\mathrm{F} 1,4.69 \pm 0.46$ for $\mathrm{F} 2$ and $7.78 \pm 1.63$ for $\mathrm{F} 3$ [22]. The obtained cutoff values are presented in table IV. The different cutoff values are explained most probably by the different ultrasound machine and transducer compared to other authors which calculated the same ratio. In the same study, Altiparmak et al, also compared ER1 with ER2 [22]. Using as the reference method, the liver biopsy, both ER were positively correlated with the degree of fibrosis but ER2 presented better results [22]. The performance and clinical usefulness of ER2 are detailed in Table IV.

6. Elastic Index (EI) was proposed by Colombo et al [24], who studied 72 patients (45 with chronic liver pathology and 27 controls). They used Hitachi Preirus ultrasound with 3.5-7 MHz linear probe and Elasto_ver 1.5.1 software for all the 10 acquired static images. The color map was converted into numbers, each color being coded from 0 to 255 . Eleven parameters were calculated from the histograms then, 4 functions (Z1-Z4) were calculated and were subsequently included in an integrative function for determining the elasticity index (see formula no 5 in appendix) [24]. Using their formula, RTE had a good accuracy to predict fibrosis and cirrhosis, but still lower than the TE and ARFI (Table V) [24].

Table IV. Elastic Ratio 2 (ER2) cutoff values and diagnostic usefulness

\begin{tabular}{|c|c|c|c|c|c|c|c|c|c|c|c|c|}
\hline Authors & Cuto & & & $\mathbf{A U C}$ & & & Se & & & $\mathrm{Sp}$ & & \\
\hline & $\mathrm{F} \geq 2$ & $\mathrm{~F} \geq \mathbf{F 3}$ & $F=4$ & $\mathrm{~F} \geq 2$ & $F \geq F 3$ & $F=4$ & $F \geq 2$ & $F \geq F 3$ & $F=4$ & $F \geq 2$ & $\mathrm{~F} \geq \mathrm{F3}$ & $F=4$ \\
\hline $\begin{array}{l}\text { Koizumi } \\
2011[18]\end{array}$ & 2.73 & 3.25 & 3.93 & 0.89 & 0.94 & 0.95 & 82.8 & 85.4 & 90.9 & 90.9 & 96.4 & 91.5 \\
\hline $\begin{array}{l}\text { Ochi } \\
2012 \text { [19] }\end{array}$ & 2.67 & 3.02 & 3.36 & 0.923 & 0.947 & 0.96 & 92.3 & 88.9 & 100 & 89.8 & 96.5 & 95.3 \\
\hline $\begin{array}{l}\mathrm{Hu} \\
2014[20]^{*}\end{array}$ & 2.62 & 3.2 & 3.86 & 0.91 & 0.93 & 0.94 & 86.2 & 91.4 & 94.1 & 88.2 & 85 & 82.8 \\
\hline $\begin{array}{l}\text { Altiparmak } \\
2015[22]^{* *}\end{array}$ & 4.28 & $\begin{array}{l}6.01 \\
(F=3)\end{array}$ & - & 0.94 & $\begin{array}{l}1 \\
(F=3)\end{array}$ & - & 92 & $\begin{array}{l}100 \\
(\mathrm{~F}=3)\end{array}$ & - & 85.3 & $\begin{array}{l}100 \\
(F=3)\end{array}$ & - \\
\hline
\end{tabular}

$\mathrm{Se}$ - sensibility; $\mathrm{Sp}$ - specificity; AUC - area under the curve; F - fibrosis stage; * The criteria for staging of chronic hepatitis was according to Chinese Program of Prevention and Cure for Viral Hepatitis (S); ** The ES was calculated using Toshiba ultrasound

Table V. Elastic Index (EI) cutoff values and diagnostic usefulness

\begin{tabular}{|c|c|c|c|c|c|c|c|c|c|c|c|c|}
\hline \multirow[t]{2}{*}{ Authors } & \multicolumn{3}{|c|}{ cutoff } & \multicolumn{3}{|l|}{ AUC } & \multicolumn{3}{|l|}{ Se } & \multicolumn{3}{|l|}{ Sp } \\
\hline & $F \geq 2$ & $\mathrm{~F} \geq \mathbf{F 3}$ & $F=4$ & $F \geq 2$ & $F \geq F 3$ & $F=4$ & $F \geq 2$ & $\mathrm{~F} \geq \mathbf{F 3}$ & $F=4$ & $F \geq 2$ & $\mathrm{~F} \geq \mathrm{F3}$ & $F=4$ \\
\hline $\begin{array}{l}\text { Colombo } \\
2012 \text { [24] }\end{array}$ & 1.89 & - & 3.6 & 0.751 & - & 0.852 & 76 & - & 80 & 65.96 & - & 90.32 \\
\hline $\begin{array}{l}\text { Wang } \\
2012[2]\end{array}$ & 55.3 & 80.7 & 90.31 & 0.92 & 0.84 & 0.66 & 81.6 & 73.1 & 71.4 & 88.2 & 75 & 80 \\
\hline
\end{tabular}

$\mathrm{Se}$ - sensibility; $\mathrm{Sp}$ - specificity; AUC- area under the curve; $\mathrm{F}$ - fibrosis stage 
Wang et al [2], used a 5-MHz transducer, in a study of 85 patients ( 55 patients with chronic viral hepatitis $B$ and 20 controls). Since image acquisition was fast enough, no artifacts were identified during patient breathing; thus, patients were asked to breathe normally during the examination. No external compression was exerted on the transducer. Measurements were performed in the right hepatic lobe, in the area where parenchymal thickness was at least $6 \mathrm{~cm}$ and intrahepatic large caliber vessels were avoided [2].

To obtain EI, Wang et al made the analysis of the same 11 parameters which were used for LFI and then, four main functions (z1-z4) were calculated and an integrative function was elaborated (see formula no 6 in appendix), in a similar manner as Colombo et al. The cutoff values are detailed in table $\mathrm{V}$.

The benefits of estimating liver stiffness with RTE are multiple: 1) RTE is a noninvasive method that allows the evaluation of a liver area larger than biopsy; 2) RTE is built in many ultrasound machines thus, during the same examination, besides the routine US assessment, the prediction of liver fibrosis is also possible; 3 ) The ROI may be placed more accurately, once the liver parenchyma is viewed in grayscale; 4) RTE is not influenced by inflammation, increased transaminases, cholestasis, congestion or even a small amount of ascites [25,26]. Last but not least, RTE is much more widely available compared to the shear wave techniques.

There are still some limitations regarding RTE. This technique was developed at first, with the intention of qualitative and semi-quantitative application for small parts and not for the abdomen. Later on, among all investigated scores, LFI was the only one recommended by one manufacturer.

The WFUMB guidelines [26] describe the optimal RTE scanning method: the patient positioned in dorsal decubitus, with the right hand above the head, breathing with normal amplitude. Patient should hold their breath during the acquisition. The probe should be placed lightly on the patient's skin, in a right intercostal space. The correct RTE image is obtained when the probe is headed to the heart, in order to detect the axial movements, useful for RTE, transmitted to the liver, and not the lateral derivate [26].

Placing the ROI is still a controversial subject. The recent guidelines recommend placing the ROI deeply inside the liver parenchyma, in order to obtain a smooth image, and a ROI size of $2.5 \times 2.5 \mathrm{~cm} \mathrm{[26].}$

In order to eliminate RTE acquisition artifacts, the operator should avoid placing the ROI next to the ribs, lungs, liver capsule, large intrahepatic vessels or too deep in the liver parenchyma [26].
Given that the literature describes multiple ways to estimate liver stiffness using RTE and also variable cutoff values, it is rather difficult to decide what is the best way to estimate liver fibrosis, keeping in mind that the result may change completely the management of the patient. The available Guidelines and Recommendations of Clinical Practice regarding RTE recommend the use of the liver stiffness index (LFI), the only method also recommended by one manufacturer for liver stiffness quantification [26].

\section{Conclusions}

At first, RTE was considered to be a simple qualitative and semi-quantitative method, since it only allowed the relative elasticity assessment of the liver. Over time, several computing methods for encoding and converting the color elasticity map into liver fibrosis grades were developed. Of all the formulas described, both the recent guidelines and one manufacturer recommend the use of the Liver Fibrosis Index (LFI) as the most accurate liver fibrosis estimation means, when using RTE.

Acknowledgement. This work was supported by a grant from the Romanian National Authority for Scientific Research and Innovation, CNCS - UEFISCDI, project number PN-II-RU-TE-2014-4-2023.

\section{Conflict of interest: none}

\section{References}

1. Friedrich-Rust M, Ong MF, Herrmann E, et al. Real-Time Elastography for Noninvasive Assessment of Liver Fibrosis in Chronic Viral Hepatitis. AJR Am J Roentgenol 2007; 188:758-764.

2. Wang J, Guo L, Shi X, Pan W, Bai Y, Ai H. Real-time elastography with a novel quantitative technology for assessment of liver fibrosis in chronic hepatitis B. Eur J Radiol 2012;81:e31-e36.

3. Regev A, Berho M, Jeffers LJ, et al. Sampling error and intraobserver variation in liver biopsy in patients with chronic HCV infection. Am J Gastroenterol 2002;97:2614-2618.

4. Bedossa P, Dargère D, Paradis V. Sampling variability of liver fibrosis in chronic hepatitis C. Hepatology 2003;38:1449-1457.

5. Bravo AA, Sheth SG, Chopra S. Liver Biopsy. N Engl J Med 2001;344:495-500.

6. Fujimoto K, Wada S, Oshita M, Kato M, Tonomura A, Mitake T. Non-invasive evaluation of Hepatic Fibrosis in patients with Chronic Hepatitis C using Elastography. Medix 2007;10 Suppl: 24-27.

7. Ziol M, Handra-Luca A, Kettaneh A, et al. Noninvasive assessment of liver fibrosis by measurement of stiffness in 
patients with chronic hepatitis C. Hepatology 2005;41:4854.

8. Castéra L, Vergniol J, Foucher J, et al. Prospective comparison of transient elastography, Fibrotest, APRI, and liver biopsy for the assessment of fibrosis in chronic hepatitis C. Gastroenterology 2005;128:343-350.

9. Foucher J, Chanteloup E, Vergniol, et al. Diagnosis of cirrhosis by transient elastography (FibroScan): a prospective study. Gut 2006;55:403-408.

10. Tatsumi C, Kudo M, Ueshima K, et al. Noninvasive evaluation of hepatic fibrosis using serum fibrotic markers, transient elastography (FibroScan) and real-time tissue elastography. Intervirology 2008;51:27-33.

11. Tatsumi C, Kudo M, Ueshima K, et al. Non-invasive evaluation of hepatic fibrosis for type $\mathrm{C}$ chronic hepatitis. Intervirology 2010;53:76-81.

12. Ferraioli G, Tinelli C, Malfitano A, et al. Performance of real-time strain elastography, transient elastography, and aspartate-to-platelet ratio index in the assessment of fibrosis in chronic hepatitis C. AJR Am J Roentgenol 2012;199:1925.

13. Fujimoto K, Kato M, Kudo M, et al. Novel image analysis method using ultrasound elastography for noninvasive evaluation of hepatic fibrosis in patients with chronic hepatitis C. Oncology 2013;84:3-12.

14. Yada N, Kudo M, Morikawa H, Fujimoto K, Kato M, Kawada N. Assessment of liver fibrosis with real-time tissue elastography in chronic viral hepatitis. Oncology 2013;84:13-20.

15. Tomeno W, Yoneda M, Imajo K, et al. Evaluation of the Liver Fibrosis Index calculated by using real-time tissue elastography for the non-invasive assessment of liver fibrosis in chronic liver diseases. Hepatol Res 2013;43:735742.

16. Meng F, Zheng Y, Zhang Q, et al. Noninvasive evaluation of liver fibrosis using real-time tissue elastography and transient elastography (FibroScan). J Ultrasound Med 2015;34:403-410.
17. Hu Y, Gong HY, Lin HJ. Real-time Tissue Elastography for Assessment of Liver Stiffness in Adults Without Known Liver Disease. J Ultrasound Med 2015;34:1895-1900.

18. Koizumi Y, Hirooka M, Kisaka Y, et al. Liver fibrosis in patients with chronic hepatitis $\mathrm{C}$ : noninvasive diagnosis by means of real-time tissue elastography - establishment of the method for measurement. Radiology 2011;258:610-617.

19. Ochi H, Hirooka M, Koizumi Y, et al. Real-time tissue elastography for evaluation of hepatic fibrosis and portal hypertension in nonalcoholic fatty liver diseases. Hepatology 2012;56:1271-1278.

20. Hu Q, Zhu SY, Kang LK, Wang XY, Lun HM, Xu CM. Non-invasive assessment of liver fibrosis using real-time tissue elastography in patients with chronic hepatitis B. Clin Radiol 2014;69:194-199.

21. Kanamoto M, Shimada M, Ikegami T, et al. Real time elastography for noninvasive diagnosis of liver fibrosis. J Hepatobiliary Pancreat Surg 2009;16:463-467.

22. Altiparmak B, Nural MS, Aydin R, Danaci M. Comparison of intrahepatic vein-to-liver parenchyma and intercostal muscle-to-liver parenchyma strain ratios in the assessment of liver fibrosis: which one should we use? Abdom Imaging 2015;40:730-737.

23. Xie L, Chen X, Guo Q, Dong Y, Guang Y, Zhang X. Realtime elastography for diagnosis of liver fibrosis in chronic hepatitis B. J Ultrasound Med 2012;31:1053-1060.

24. Colombo S, Buonocore M, Del Poggio A, et al. Head-tohead comparison of transient elastography (TE), real-time tissue elastography (RTE), and acoustic radiation force impulse (ARFI) imaging in the diagnosis of liver fibrosis. J Gastroenterol 2012;47:461-469.

25. Hirooka M, Koizumi Y, Hiasa Y, et al. Hepatic elasticity in patients with ascites: evaluation with real-time tissue elastography. AJR Am J Roentgenol 2011;196:W766-W771.

26. Ferraioli G, Filice C, Castera L, et al. WFUMB guidelines and recommendations for clinical use of ultrasound elastography: Part 3: Liver. Ultrasound Med Biol 2015;41:11611179. 